\title{
Method of increasing durability of thin-walled structural elements
}

\author{
Vladimir Mironov ${ }^{1,2}$, Olga Lukashuk $^{2, *}$, and Dmitriy Ogorelkov ${ }^{2}$ \\ ${ }^{1}$ Institute of Engineering Science, RAS (Ural Branch), Yekaterinburg, Russia \\ ${ }^{2}$ Ural Federal University, Yekaterinburg, Russia
}

\begin{abstract}
The article analyzes how efficient damping introduced into designs of structural elements by means of such light fillers as foam plastics could be. It cites the results of computing experiments designed to assess how the number of significant free vibration cycles depends on the width of a beam wall and the height of a filler layer. The prediction is made of a substantial increase in durability of the machines which operate in a dynamic impulse-loading mode.
\end{abstract}

\section{Introduction}

The problem (common for many branches of technology) of extending the life of a product is dealt with using different approaches. Most often, they are about modifying that surface layer of the product where fatigue cracks tend to appear [1]. For thin-walled structural elements, such approaches have limited application.

On the other hand, the increase in durability could be achieved by decreasing dynamics of machines and constructions - for example, by means of different vibration dampers [2]. In building and aircraft construction, those goals have long and quite successfully been realized through the usage of threelayer plates and shells [3].

Box beams and thin-walled closed-profile rods are widely utilized in bearing metallic structures of mining and transporting machines. During their normal operation, such machines are subject to a wide range of regular or random dynamic loads.

The decrease in dynamics helps to extend the life of such machines and improve the working conditions for their operators. The usage of such light-density fillers as foam plastics - though leading to some insignificant increase in product weight - provide a substantial gain in the protection of personnel (from noise and harmful vibration) and structures (from corrosion). But the main purpose of such a filler capable of absorbing the energy of vibrations is to reduce their number and amplitude when external dynamic loads are applied.

The goal of the work is to make preliminary assessment on practicability of using artificial lowmodulus elastic materials to damp thin-walled elements of metallic structures. The task is realized via conducting computing experiments in the environment of the SolidWorks software.
Estimating bending stresses turns out to be a crucial factor in assessing strength and durability of most elements pertaining to bearing structures of machines. Therefore, the research is limited to free vibration of beams subject to bending. To achieve that specific goal, the following tasks were realized:

- calculating the number of significant damped free vibration cycles;

- parametrically analyzing a cantilever beam;

- parametrically analyzing an overhead crane bridge girder.

\section{Calculation of the number of significant free vibration cycles for a beam}

Evaluative calculations justify analyzing vibrations of a beam as a material point of a reduced mass $m_{p}$ on a spring of an equivalent rigidity $c_{e}$. Free vibrations of such a point are described by homogeneous differential equations of the second order [4]. Neglecting resistance forces, we have

$$
\ddot{x}+p^{2} x=0,
$$

where $p^{2}=c_{e} / m_{p}$ - square of angular frequency, $c_{e}-$ rigidity of a steel beam, $m_{p}$ - reduced rigidity of a beam without a filler. The solution of equation (1) describes free harmonic vibrations of a frequency $\omega=p / 2 \pi$ and a period $T=1 / \omega$.

When a system is affected by low resistance forces proportional to a first degree speed of motion $R=\alpha x-$ which is the case, for example, for building structures we have

\footnotetext{
Corresponding author: oldim96@mail.ru
} 


$$
\ddot{x}+2 n \dot{x}+p_{1}^{2} x=0,
$$

where $n=\alpha / 2 m_{p r}, \alpha$ - factor of resistance, $m_{p r}-$ reduced mass of a beam with a filler, $p_{1}^{2}=c_{e r} / m_{p r}-$ square of angular frequency for a beam with a filler, $c_{e r}$ - equivalent rigidity of a beam with a filler. The solution of equation (2) describes damped vibrations of a frequency $\omega_{1}=p_{1} / 2 \pi$ and a conditional period $T_{1}=2 \pi / p_{1}$. The angular frequency of free damped vibrations is determined by the expression

$$
p_{1}=\sqrt{p^{2}-n^{2}}
$$

Starting conditions to determine the integration constants in equations (1) and (2) are set to be the same as the ones realized in the follow-up experiment: for $t=0$, we have $x=x_{0} ; \dot{\mathrm{x}}_{0}=0$.

The solution of equation (2) describes, for $n<p_{1}$, damped beam vibrations with a gradually diminishing amplitude. The envelopes of the process curve are determined by the functions

$$
x= \pm x_{0} e^{-n t} .
$$

Significant vibration amplitudes, regardless of an initial displacement $x_{0}$, are estimated by the relation $x / x_{0} \geq 0,1$. Then, the corresponding time $t_{*}$ could be determined through formulas (3) and (4)

$$
t_{*}=-\frac{\ln 0,1}{n}=-\frac{\ln 0,1}{\sqrt{p^{2}-p_{1}^{2}}} .
$$

The predicted number of significant cycles $\mathrm{N}$ is estimated by

$$
N=\frac{t_{*}}{T_{1}}=-\frac{p_{1} \ln 0,1}{2 \pi \sqrt{p^{2}-p_{1}^{2}}}=-\frac{\omega_{1} \ln 0,1}{2 \pi \sqrt{\omega^{2}-\omega_{1}^{2}}}
$$

and could be determined for known frequencies $p$ and $p_{1}$. Modern computer systems allow to find natural frequencies $\omega, \omega_{1}$ for thin-walled elements of any structure.

Based on the results of energy approach to assessment of damping properties of a structure, we select the width $S$ of the wall and the height $h$ of the foam plastic layer in a beam as variable parameters.

Frequency analysis for a cantilever beam in its initial state and with a foam plastic filler is made using the SolidWorks software environment [5].

Estimating the damping effect which foam plastics produce using formula (6), suppose that $\omega$ is the natural frequency of a beam without a filler, and $\omega_{1}$ is the one of a beam with a filler.

\section{Parametric analysis of test cantilever beams}

For the purposes of conducting the experiment, it was decided to use steel test beams of $l=600 \mathrm{~mm}$ in length, $b$ $=25 \mathrm{~mm}$ in box-section width, $H=70 \mathrm{~mm}$ in height and $S=1,5 \mathrm{~mm}$ in wall thickness. To carry out the frequency analysis, a solid model for a box beam and an assembly model filled with a foam plastic material were built. Lowest vibration frequencies of beams in their initial state or with the foam plastic filler were determined for the interval $S \in[0,2 ; 1,5] \mathrm{mm}$.

The results of the numerical experiment are presented in Fig. 1 as relations between the number of significant vibration cycles $N$ and the thickness of the filled-beam wall $S$.

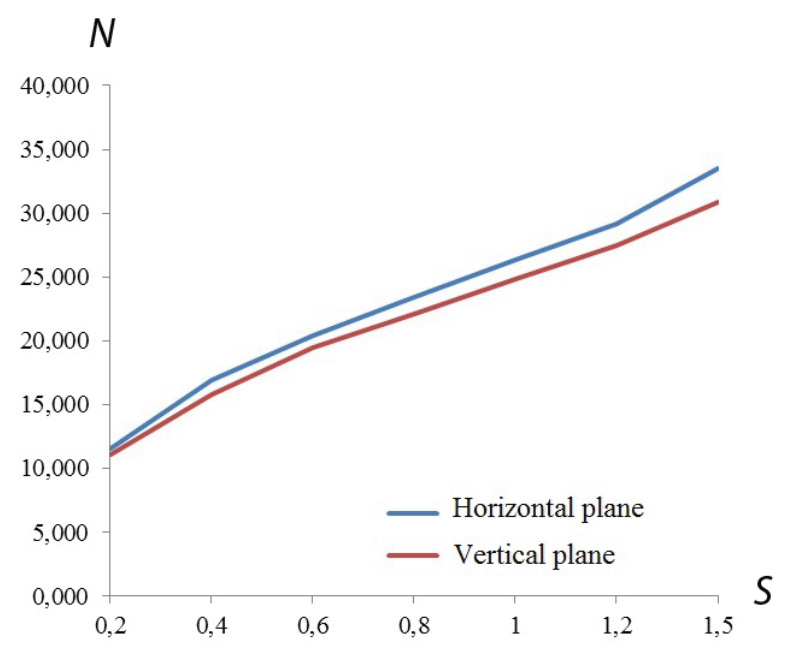

Fig. 1. The number of significant vibrations in relation to the thickness of the beam section wall.

Reference sources cite data on logarithmical decrements of metallic bridge structures in the range of $\delta=0,05 \ldots 0,12$. For the given significance level of 0,1 , the number of free vibrations is within 49-19 cycles. In accordance to the graphs in Fig. 1, the number of vibrations is 33-11 cycles, which is approximately $30 \%$ lower than in the case of the values for the box beam. This result should be considered as a significant one. The obtained data on the predicted number of cycles for a filled beam pass validation tests.

\section{Parametric analysis of an overhead crane bridge girder}

Calculations for the design of an overhead bridge crane of 20 tons in lifting capacity and 19,5 meters in span are made using the procedure described in the tutorial [6]. On the condition of structural rigidity, the following cross-sectional dimensions were chosen for the bridge girder: $H=1,1 \mathrm{~m}, b=0,6 \mathrm{~m}, S=9 \mathrm{~mm}$.

Fig. 2 shows the form of bending vibrations for the girder assumed to be fastened on specific selected conditions. 


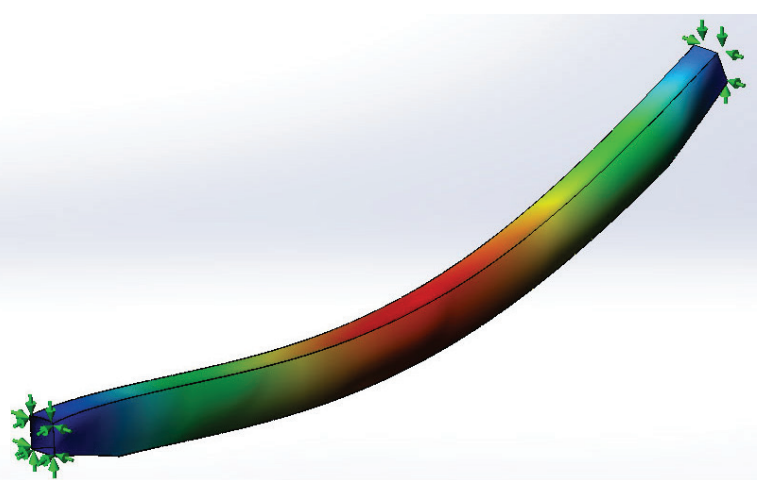

Fig. 2. The form of bending vibrations for the bridge girder of the crane.

The logarithmical decrement of vibrations $\delta$ is related to the factor of damping $n$ - which is determined from expression (3) - as $\delta=T_{1} n$. Its values are often given in reference sources. Tables 1 and 2 provide the decrement values predicted for bridge girders with various heights of the filler layer $H$.

Table 1. Logarithmical decrement of free damped vibrations $\delta$ in the horizontal plane.

\begin{tabular}{|c|c|c|c|c|c|}
\hline \multirow{2}{*}{$\begin{array}{c}\text { Wall } \\
\text { thickne } \\
\text { ss, } \boldsymbol{m m}\end{array}$} & \multicolumn{5}{|c|}{ Filler layer height, $\boldsymbol{m m}$} \\
\cline { 2 - 6 } & 220 & 440 & 660 & 880 & 1100 \\
\hline 4 & 0,158 & 0,176 & 0,190 & 0,207 & 0,231 \\
\hline 5 & 0,134 & 0,152 & 0,168 & 0,182 & 0,207 \\
\hline 6 & 0,116 & 0,134 & 0,151 & 0,168 & 0,189 \\
\hline 7 & 0,091 & 0,109 & 0,128 & 0,145 & 0,174 \\
\hline 8 & 0,070 & 0,094 & 0,115 & 0,129 & 0,160 \\
\hline 9 & 0,053 & 0,081 & 0,100 & 0,113 & 0,146 \\
\hline 10 & 0,049 & 0,074 & 0,094 & 0,109 & 0,137 \\
\hline
\end{tabular}

Table 2. Logarithmical decrement of free damped vibrations $\delta$ in the vertical plane.

\begin{tabular}{|c|c|c|c|c|c|}
\hline \multirow{2}{*}{$\begin{array}{c}\text { Wall } \\
\text { thickne } \\
\text { ss, } \boldsymbol{m m}\end{array}$} & \multicolumn{5}{|c|}{ Filler layer height, $\boldsymbol{m m}$} \\
\cline { 2 - 6 } & 220 & 440 & 660 & 880 & 1100 \\
\hline 4 & 0,124 & 0,173 & 0,203 & 0,233 & 0,251 \\
\hline 5 & 0,099 & 0,145 & 0,173 & 0,197 & 0,222 \\
\hline 6 & 0,089 & 0,125 & 0,152 & 0,177 & 0,194 \\
\hline 7 & 0,081 & 0,127 & 0,149 & 0,169 & 0,184 \\
\hline 8 & 0,076 & 0,111 & 0,137 & 0,153 & 0,171 \\
\hline 9 & 0,067 & 0,100 & 0,122 & 0,143 & 0,158 \\
\hline 10 & 0,065 & 0,098 & 0,118 & 0,133 & 0,152 \\
\hline
\end{tabular}

Fig. 3 shows graphs for the decrement change in relation to the parameters selected. The damping capacity of a structure decrease with an increase in the wall thickness and a decrease in the filler layer height.
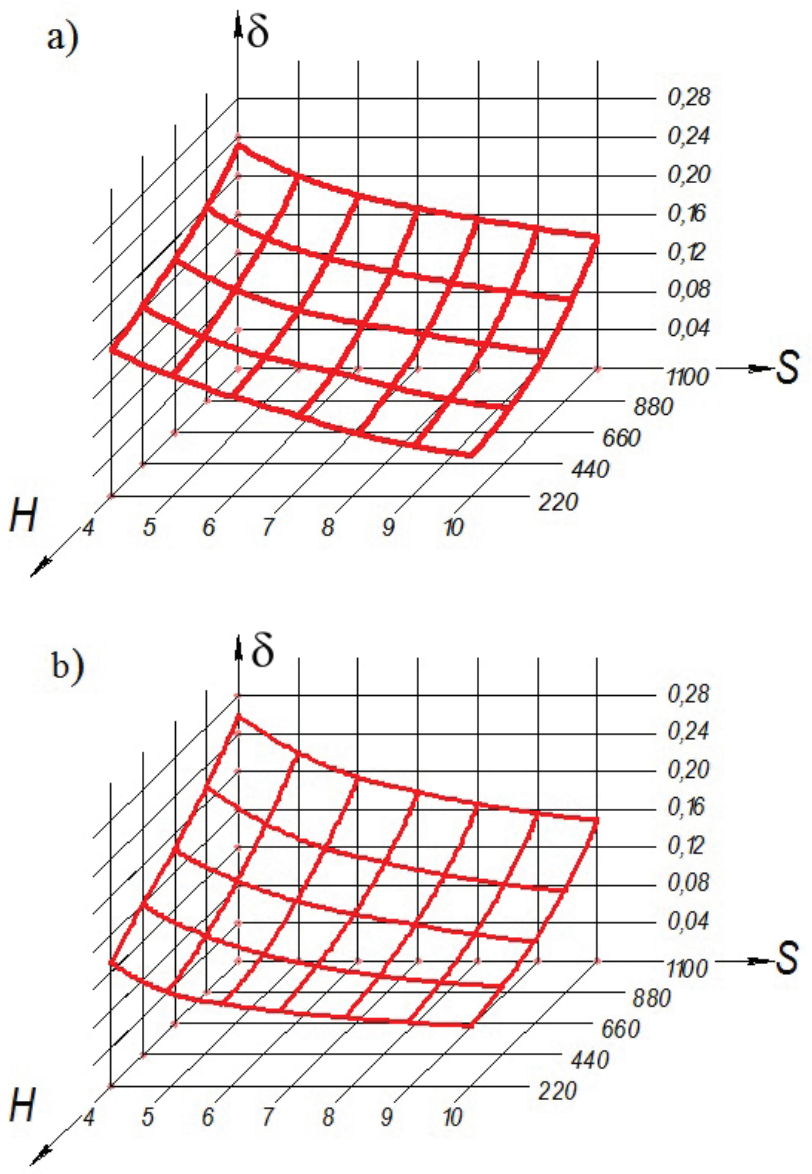

Fig. 3. Relation of the logarithmical decrement of damping to the wall thickness and the foam plastic layer height in the horizontal a) and vertical b) planes.

Similarly, the number of significant cycles also decreases (Tables 3 and 4). Graphs for the relation of the number of cycles to the girder wall thickness and the damping layer height for the given intervals are provided by Fig. 4.

Table 3. The number of significant cycles $N$ in the horizontal plane.

\begin{tabular}{|c|c|c|c|c|c|}
\hline \multirow{2}{*}{$\begin{array}{c}\text { Wall } \\
\text { thickne } \\
\text { ss, } \boldsymbol{m m}\end{array}$} & \multicolumn{5}{|c|}{ Filler layer height, $\mathbf{m m}$} \\
\cline { 2 - 6 } & 220 & 440 & 660 & 880 & 1100 \\
\hline 4 & 14,604 & 13,093 & 12,096 & 11,122 & 9,964 \\
\hline 5 & 17,121 & 15,175 & 13,717 & 12,634 & 11,125 \\
\hline 6 & 19,876 & 17,152 & 15,200 & 13,731 & 12,162 \\
\hline 7 & 25,287 & 21,059 & 17,951 & 15,852 & 13,246 \\
\hline 8 & 32,899 & 24,509 & 19,946 & 17,791 & 14,435 \\
\hline 9 & 43,417 & 28,508 & 23,063 & 20,380 & 15,729 \\
\hline 10 & 47,454 & 31,003 & 24,495 & 21,045 & 16,764 \\
\hline
\end{tabular}


Table 4. The number of significant cycles $N$ in the vertical plane.

\begin{tabular}{|c|c|c|c|c|c|}
\hline \multirow{2}{*}{$\begin{array}{c}\text { Wall } \\
\text { thickne } \\
\text { ss, } \boldsymbol{m m}\end{array}$} & \multicolumn{5}{|c|}{ Filler layer height, $\boldsymbol{m m}$} \\
\cline { 2 - 6 } & 220 & 440 & 660 & 880 & 1100 \\
\hline 4 & 18,515 & 13,093 & 11,365 & 9,883 & 9,157 \\
\hline 5 & 23,239 & 15,175 & 13,302 & 11,705 & 10,382 \\
\hline 6 & 25,777 & 17,152 & 15,184 & 12,995 & 11,863 \\
\hline 7 & 28,429 & 21,059 & 15,468 & 13,643 & 12,499 \\
\hline 8 & 30,130 & 24,509 & 16,773 & 15,064 & 13,487 \\
\hline 9 & 34,401 & 28,508 & 18,876 & 16,158 & 14,530 \\
\hline 10 & 35,625 & 31,003 & 19,588 & 17,303 & 15,110 \\
\hline
\end{tabular}
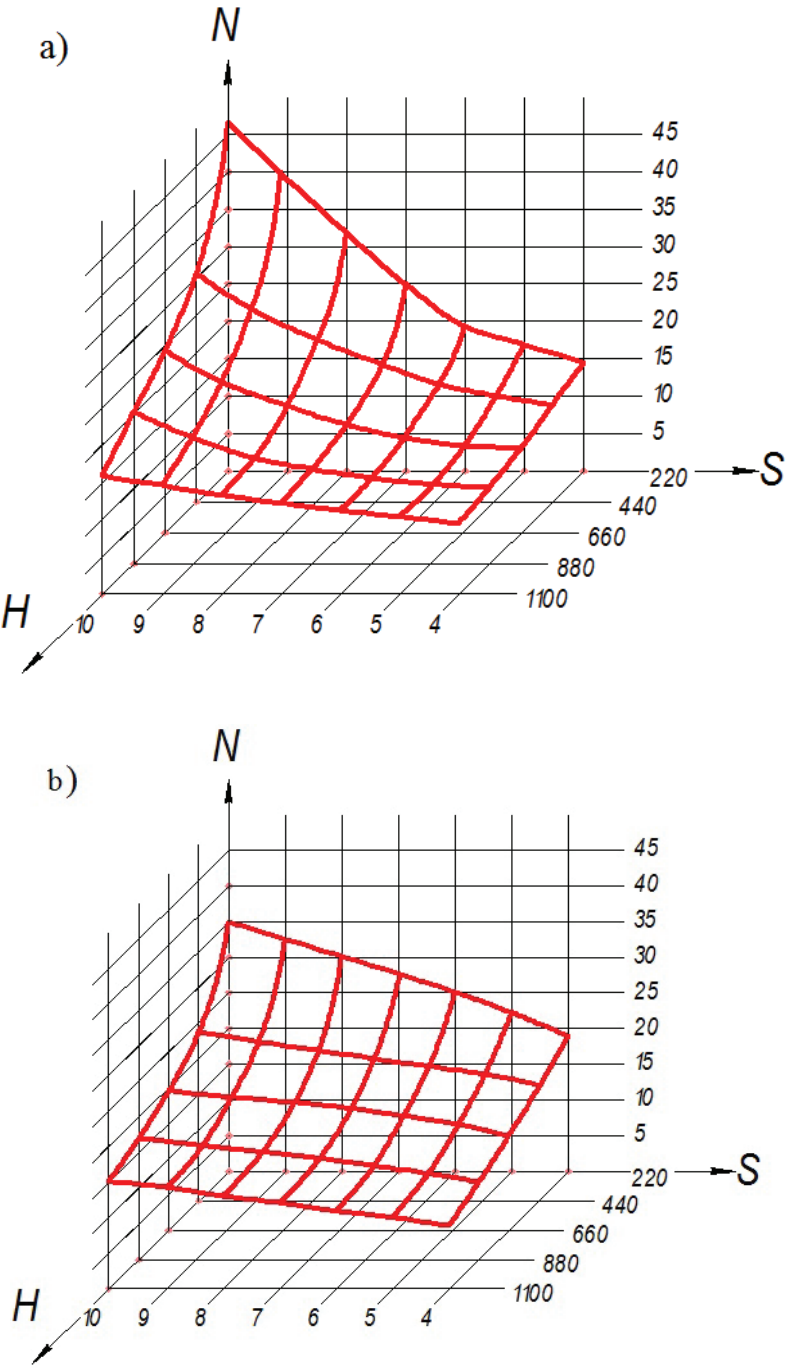

Fig. 4. The number of significant vibrations for the bridge girder in the horizontal a) and vertical b) planes.

Both for the laboratory-tested beam in Fig. 1 and the bridge girder in Fig. 4, the prediction is made of getting major damping effect from using light low-modulus foam plastics as fillers. Thus, for the calculated wall thickness of $9 \mathrm{~mm}$, an increase in the filler layer height led to a decrease in the number of the bridge girder vibrations from 43 to 16 in the horizontal plane and from 34 to 14 in the vertical one (see Tables 3 and 4). The filler does not impact the selection of the girder section, which is determined by the conditions of structural strength and rigidity. But it does improve the recommendation given on the maximum possible decrease in the wall thickness.

The decrease in the number of significant cycles predicted for metallic structures damped by means of low-modulus foam plastics would provide the basis for a very efficient method of extending the life of machines. And, particularly, it is true for mining, lifting, carrying, road-building machines, which are subject, during their operation, to a wide range of dynamic loads. A more founded conclusion could be provided by conducting tests on steel beams under repeatedly varied impulse loading.

\section{Conclusion}

Computing experiments revealed only a possibility in principle of extending the life of machine parts damped by a filler. It is necessary to check directly in experiments the fatigue properties of the filler, its adhesion to metal. It would be useful to further study the layout of positioning the filler within a structure in order to find a rational solution. Assessment of the project cost is also needed to define optimization problems. At the same time, the provided results showed that such a method of damping has good prospects in terms of increasing the durability of structural elements.

\section{References}

1. A.M. Shhipachev, V.S. Muhin, Izv. vuzov. Aviacionnaja tehnika, 1, 76 (2002)

2. Ya.L. Liberman, K.Yu. Letnev, Automated Vibration Elimination for the Load Lifted or Lowered on a Flexible Suspension (UrFU, Ekaterinburg, 2012)

3. V.V. Bolotin, Yu.N. Novichkov, Mechanics of Multilayer Structures (Mashinostroenie, Moscow, 1980)

4. V.I. Smirnov, Course on Higher Mathematics (Nauka, Moscow, 1974, 2)

5. A.A. Aljamovskij, A.A. Sobachkin, E.V. Odincova, etc., Computer-aided Design in SolidWorks for Engineering Purposes (BHV-Peterburg, Saint Petersburg, 2005)

6. V.E. Dus'e, Yu.V. Navarskij, V.P. Zhegul'skij, Calculation and Design of Bridge Crane Metallic Structures (UGTU-UPI, Ekaterinburg, 2007) 